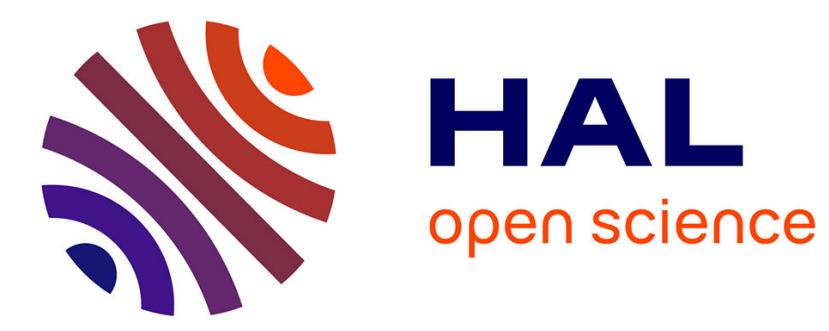

\title{
Magnetorelaxometry of Nanoparticles Using a GMI Magnetometer
}

Basile Dufay, Sébastien Saez, Matthieu Denoual, Christophe Dolabdjian, F. Ludwig, E. Heim, M. Schilling, L. Melo, A. Yelon, D. Menard

\section{- To cite this version:}

Basile Dufay, Sébastien Saez, Matthieu Denoual, Christophe Dolabdjian, F. Ludwig, et al.. Magnetorelaxometry of Nanoparticles Using a GMI Magnetometer. Sensor letters, 2010, 7 (3), p429-432. 10.1166/sl.2009.1087 . hal-00980166

\section{HAL Id: hal-00980166 https://hal.science/hal-00980166}

Submitted on 7 May 2014

HAL is a multi-disciplinary open access archive for the deposit and dissemination of scientific research documents, whether they are published or not. The documents may come from teaching and research institutions in France or abroad, or from public or private research centers.
L'archive ouverte pluridisciplinaire $\mathbf{H A L}$, est destinée au dépôt et à la diffusion de documents scientifiques de niveau recherche, publiés ou non, émanant des établissements d'enseignement et de recherche français ou étrangers, des laboratoires publics ou privés. 


\title{
Magnetorelaxometry of Nanoparticles Using a GMI Magnetometer
}

\author{
B. Dufay ${ }^{1}$, S. Saez ${ }^{1}$, M. Denoual1, *, C. Dolabdjian¹, F. Ludwig², E. Heim², M. Schilling², \\ L. Melo ${ }^{3}$, A. Yelon ${ }^{3}$, and D. Menard ${ }^{3}$ \\ ${ }^{1}$ GREYC (CNRS - UMR6072), ENSICAEN and Université de Caen Basse Normandie, \\ 6 boulevard Maréchal Juin, 14050 Caen cedex, France \\ ${ }^{2}$ Institut für Elektrische Messtechnik und Grundlagen des Elektrotechnik, TU Braunschweig, \\ Hans-Sommer-Str. 66, D-38106 Braunschweig, Germany \\ ${ }^{3}$ École Polytechnique de Montréal, Département de génie physique and Regroupement québécois des matériaux \\ de pointe, CP 6079, succursale Centre-ville, Montréal, Que., H3C 3A7, Canada
}

\begin{abstract}
We have performed magnetorelaxometry measurements on superparamagnetic magnetite $\left(\mathrm{Fe}_{3} \mathrm{O}_{4}\right)$ nanoparticles using a low-cost custom-made Giant Magnetolmpendance magnetometer. We demonstrate that we are able to evaluate the concentration of nanoparticles in the sample (with a volume of around $150 \mu \mathrm{l}$ ) by comparison with reference measurements of the induced magnetic field using fits to the Néel expression for magnetorelaxation.
\end{abstract}

Keywords: GMI Magnetometer, Magnetorelaxometry, Magnetic Nanoparticles (MNPS).

\section{INTRODUCTION}

Magnetic nanoparticles are increasingly studied for biological and medical applications. They are used as markers, for biomaterial manipulation and drug delivery in targeted therapy, ${ }^{1-3}$ and even for treatment through magnetic heating. ${ }^{4}$ As markers, they are not susceptible to bleaching as are fluorescent dyes, are non-toxic, and allow detection in opaque media. Making use of these advantages, magnetic immunoassays have been developed to quantify biological targets. The so-called magnetic relaxation immunoassay (MARIA) is based on the magnetic relaxation of magnetic nanoparticles. ${ }^{5}$ The Magnetorelaxation technique, first proposed 13 years ago, has since been widely developed.

The first magnetorelaxometry measurements were carried out using SQUID sensors. ${ }^{6}$ The SQUID is known to be one of the most sensitive solid state magnetic field sensors, but it requires liquid helium or liquid nitrogen cooling and is limited in terms of picking up the initial part of the relaxation cycle. More recently, a similar system using room temperature fluxgate magnetometers has been developed. ${ }^{7}$ These can be positioned closer to the sample,

${ }^{*}$ Corresponding author; E-mail: mdenoual@greyc.ensicaen.fr partially compensating for their lower intrinsic sensitivity and are able to pick up the entire relaxation cycle.

The giant magnetoimpedance (GMI) effect ${ }^{8,9}$ has been proposed for biosensing. ${ }^{10} \mathrm{New}$ developments in high sensitivity magnetic sensors ${ }^{11,12}$ show the potential of GMI magnetometers to improve over fluxgate magnetometers, ${ }^{7}$ at lower cost. This paper demonstrates the use of a custom made low-cost GMI magnetometer to perform magnetorelaxometry measurements on dilutions of magnetite nanoparticles. We first present the characteristics of the GMI magnetometer which we have developed, and its implementation in a magnetorelaxometry measurement system. We then demonstrate the detection of the relaxation behavior of magnetic nanoparticles, using the magnetorelaxometry technique.

\section{PRINCIPLE OF OPERATION}

Magnetorelaxometry is based on the measurement of the magnetic signal of superparamagnetic nanoparticles under an external magnetic field. When the field is turned on, the magnetic moments of the nanoparticles are aligned and the sample shows a measurable signal (see Fig. 1), proportional to the nanoparticle magnetization $m_{\mathrm{MNPs}}$. This 


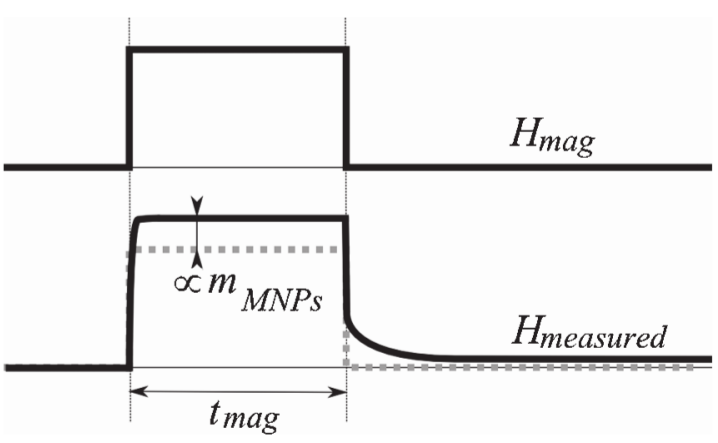

Fig. 1. External applied magnetic field and sensed magnetic signal in a magnetorelaxometry experiment.

signal depends upon the applied external field, the magnetic properties of the nanoparticles, their distance from the magnetometer and their concentration. When the external magnetic field is abruptly turned off, the magnetic moments of the sample of nanoparticles relax according to different mechanisms, depending upon whether the nanoparticles are mobile or immobilized. ${ }^{13}$ For immobilized nanoparticles, the magnetization decays only via the Néel mechanism. ${ }^{7}$ Mobile nanoparticles also relax through Brownian motion. By measuring the magnetization decay, the degree to which the nanoparticles are bound can be distinguished by their different relaxation behavior. They can then be used as biomarkers when functionalized with specific biomaterials, such as antibodies or antigens.

\section{EXPERIMENTAL DETAILS}

Magnetorelaxometry measurements were performed on a series of six $150 \mu \mathrm{l}$ samples of dilute magnetite $\left(\mathrm{Fe}_{3} \mathrm{O}_{4}\right)$ superparamagnetic nanoparticles. Each new sample is diluted by a factor of two with DI water from the previous one, from $\mathrm{N} 1$ being the most concentrated with an Fe content of $22.9 \mathrm{mg} / \mathrm{ml}$, to $\mathrm{N} 6$, the least concentrated (diluted by a factor of 32). The nanoparticles were immobilized by freeze-drying, so that the sample magnetization decays via the Néel mechanism.

The experimental set-up is similar to the fluxgate magnetorelaxometry system developed by Ludwig et al. ${ }^{14}$ The GMI magnetometer, $3 \mathrm{~cm}$ long and $1 \mathrm{~cm}$ wide, is located near the center of a Helmholtz coil with its sensitive axis perpendicular to the applied field, minimizing the signal in the absence of a sample. The sample containing the magnetic nanoparticles is positioned adjacent to one end of the magnetometer, in practice 2 to 4 millimeters, so that the signal is maximized. The Helmholtz coil produces a magnetic induction $B_{\mathrm{mag}}(1 \mathrm{mT}$ to $2 \mathrm{mT})$ during a time interval $t_{\text {mag }}(0.4 \mathrm{~s}$ to $5 \mathrm{~s})$ aligning the particles'magnetic moments. After switch-off of the magnetic field, random scattering induces a small relaxation of the sensed magnetic signal. For the relaxation processes to be observable, the magnetic field must be switched off rapidly. For this purpose, a voltage amplifier and drive electronics, providing a linear decay of the magnetic field within $400 \mu \mathrm{s}$, were constructed. The current flowing through the Helmholtz coil was determined using a resistor placed in series with it. The signal was low-pass filtered $(10 \mathrm{kHz})$, amplified, and averaged several times to improve the signal-to-noise-ratio. All measurements were performed at room temperature within the GREYC magnetic shielded room (one layer in soft iron, four in $\mu$-metal, one in aluminum, white noise $<20 \mathrm{fT} / \sqrt{\mathrm{Hz}}$ ).

The GMI magnetometer used a CoFeSiBNb soft magnetic wire, with ac and dc bias and a peak detector ${ }^{12,15}$ along with an integrator, a resistor and a feedback coil. In this locked loop mode, the GMI device operates around an optimal working point. The magnetic wire used yielded an equivalent magnetic noise spectral density of $25 \mathrm{pT} / \sqrt{\mathrm{Hz}}$ at $10 \mathrm{kHz}$ (values down to $3 \mathrm{pT} / \sqrt{\mathrm{Hz}}$ were previously reported in a similar design with a better sensing element). ${ }^{12}$ The measured values for sensitivity, band-width and dynamic range were $90 \mathrm{kV} / \mathrm{T}$, more than $10 \mathrm{kHz}$, and $130 \mathrm{~dB} / \sqrt{\mathrm{Hz}}$, respectively. The expected slew-rate is $22 \mathrm{~T} / \mathrm{s}$ (a value of $1.4 \mathrm{~T} / \mathrm{s}$ is measured, corresponding to the Helmholtz coil excitation decay). While the equivalent magnetic noise is limited by the GMI wire, the other characteristics of the magnetometer are limited by the feedback electronics.

\section{RESULTS}

Figure 2 shows the relaxation curves, averaged 128 times, measured for the three most concentrated freeze-dried magnetic nanoparticle samples. The samples were magnetized in a magnetic induction of $2 \mathrm{mT}$ during $2.5 \mathrm{~s}$.

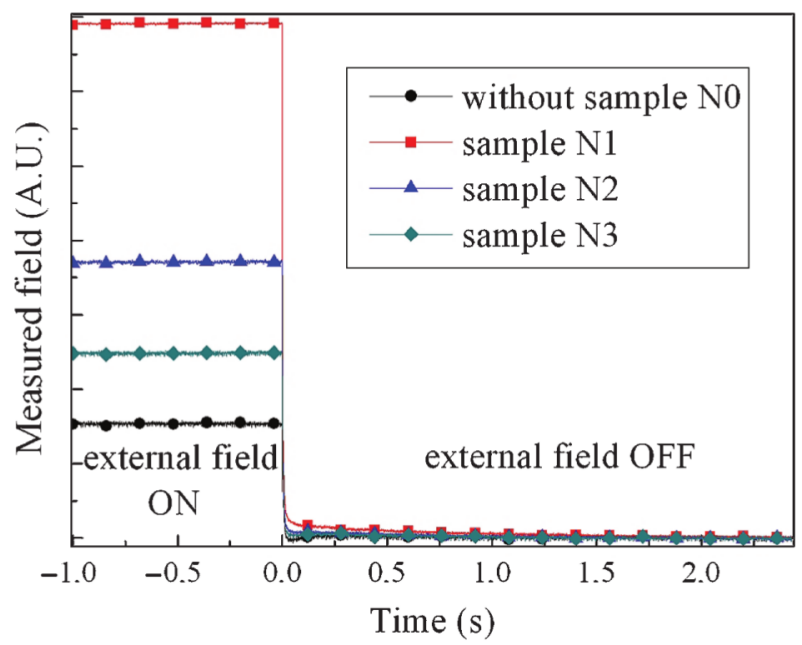

Fig. 2. Magnetic relaxation signal of freeze-dried magnetite samples, measured with a GMI magnetometer, and averaged 128 times. N1 corresponds to the most concentrated sample and N0 to the measurement without sample. To account for drifts of the system offset, the individual curves were offset-corrected to zero at $t=2.5 \mathrm{~s}$. An external magnetic induction of $2 \mathrm{mT}$ was applied for $2.5 \mathrm{~s}$. 


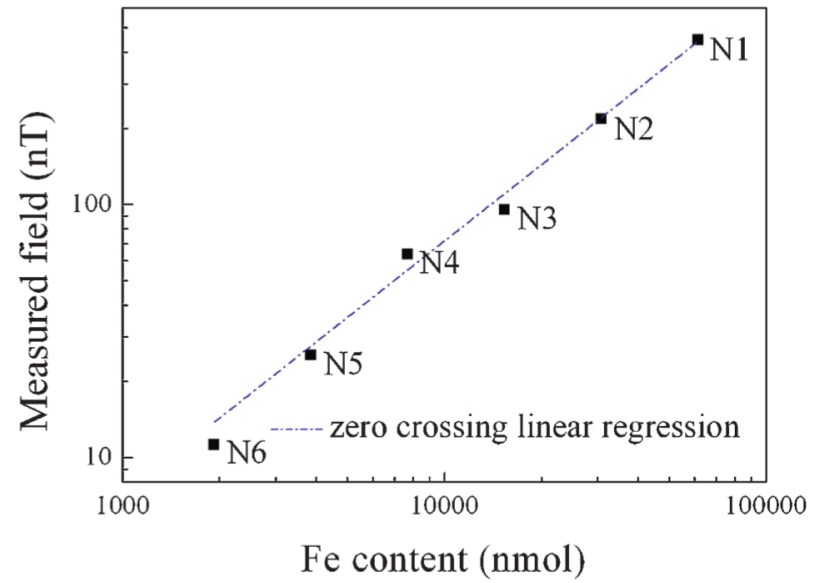

Fig. 3. Measured field versus sample concentration of Fe in the sample with zero crossing linear regression (dotted line). An external magnetic induction of $1 \mathrm{mT}$ was applied for $2 \mathrm{~s}$. Measured curves were averaged 16 times.

A non-zero response without the sample, likely due to the non-orthogonality between the Helmholtz coil axis and the GMI magnetometer, was initially observed. To compensate for this misalignment and for the equivalent magnetic field drift of the measuring system, measurement on each sample was systematically preceded by a measurement without sample. The difference between each pair of results yielded a signal proportional to the magnetization of the corresponding sample. Figure 3 shows the measured stray field of the magnetized samples as a function of their concentration. The signals exhibit near linear variation with sample concentration, as expected.

Let us now focus on the relaxation when the external field is turned off. The signal decay caused by Néel relaxation could be clearly observed for the most concentrated samples, N1 and N2, as shown in Figure 4. The signal without sample was subtracted from that with sample. The experimental relaxation curves of immobilized superparamagnetic nanoparticles, after the initial transition, can be fitted well by the expression ${ }^{11}$

$$
B=B_{\text {off }}+B_{\mathrm{N}} \ln \left(1+\frac{\tau_{\mathrm{N}}}{t-t_{0}}\right)
$$

where $B_{\text {off }}, B_{\mathrm{N}}$ and $\tau_{\mathrm{N}}$ are fit parameters, $t_{0}$ is the switchoff time of the magnetizing field. The value of $\tau_{\mathrm{N}}$ and $t_{0}$ were determined with the fit of the most concentrated sample, N1, and then used as a constant to fit samples N2 to N4. The result is shown in Figure 4. The Néel amplitude $B_{\mathrm{N}}$ extracted from the fits, is plotted as a function of the magnetic nanoparticle content of the sample (in Fig. 5). We observe the expected linear behavior. ${ }^{7}$ Due to the low signal-to-noise ratio, fits for the least concentrated samples could not be performed.

Improvement in the mechanical adjustment of the setup and reduction of the GMI magnetometer noise to levels lower than or comparable to that of fluxgates

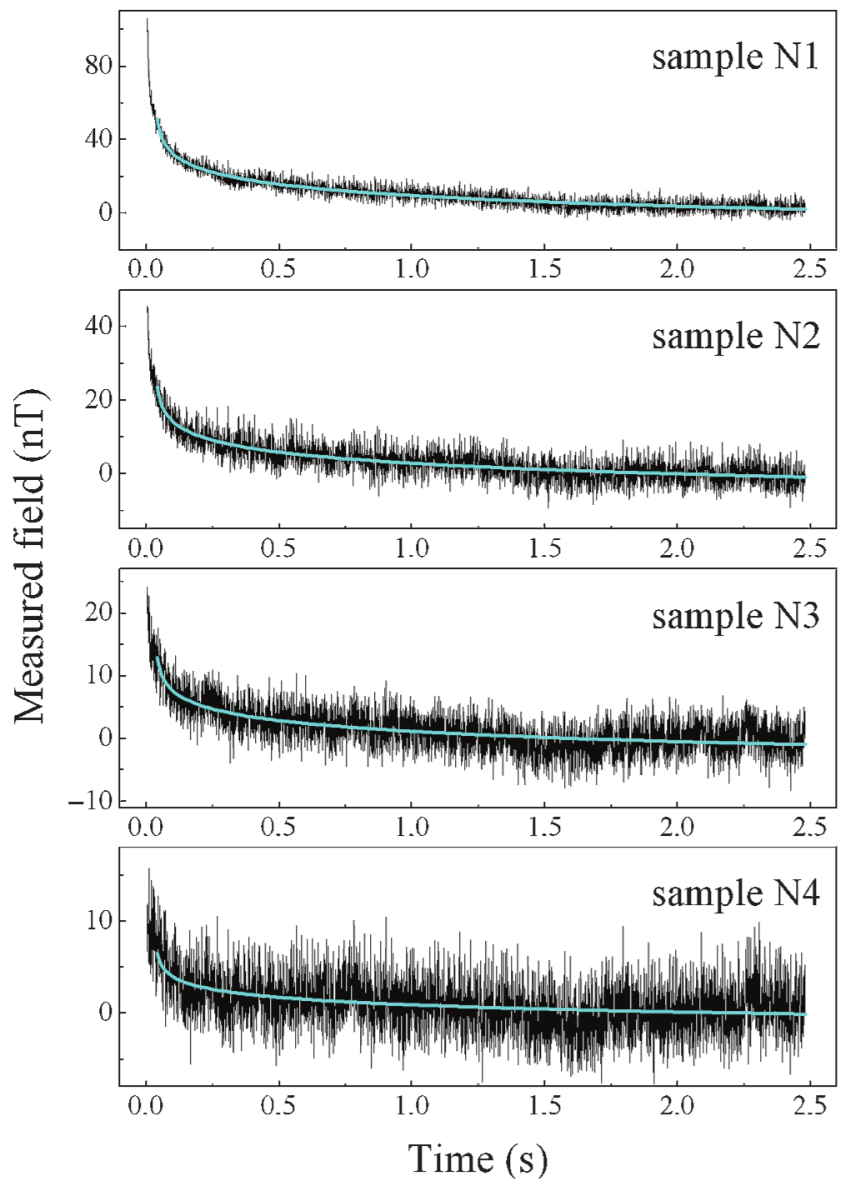

Fig. 4. Magnetorelaxometry signals measured on a dilution series of freeze-dried magnetite samples with their fit to the Néel expression of relaxation (1). The signal measured without a sample was subtracted from the measured relaxation curves before fitting. All curves were averaged 128 times.

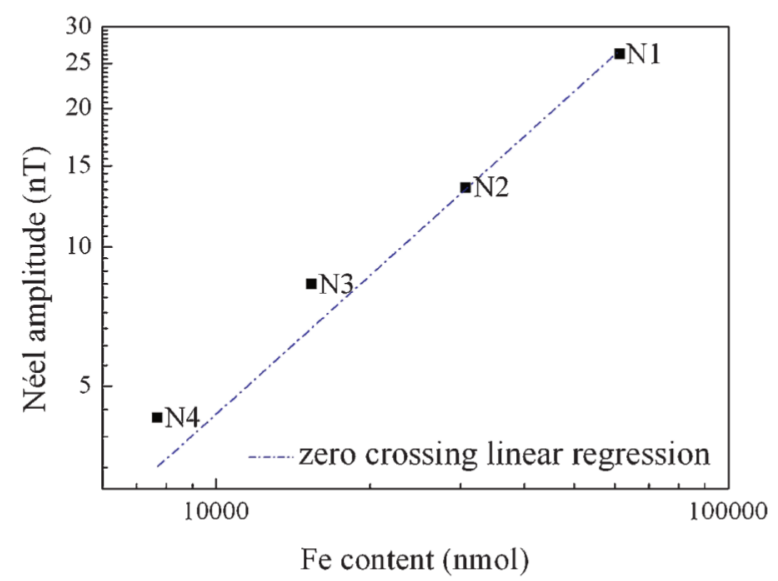

Fig. 5. Néel amplitude obtained from fitting the measured relaxation curves with Eq. (1) versus iron concentration of the sample, with zero crossing linear regression (dotted line). 
(few $\mathrm{pT} / \sqrt{ } \mathrm{Hz}$ ) are required for the extraction of the Néel amplitude for those samples. The high dynamic range, the high bandwidth and the high slew-rate of the GMI magnetometer allows for an improvement of measurements without the low temperature operation required for SQUID-based systems. Compared to fluxgates, GMI magnetometers should be less expensive and more compact. However, some optimization is still required to take advantage of their full potential. The size of the magnetic wire core of the GMI magnetometer, several tens of $\mu \mathrm{m}$ in diameter, could provide an advantage for integration in biomicrosystems.

\section{CONCLUSIONS}

We have demonstrated that the GMI magnetometers are suitable for magnetorelaxometry experiments on superparamagnetic magnetite $\left(\mathrm{Fe}_{3} \mathrm{O}_{4}\right)$ nanoparticles. As expected from theory, the Néel amplitude extracted from the fits is proportional to the concentration of the magnetic nanoparticles in the samples. The signal-to-noise ratio of the GMI magnetometers used in those experiments limited the detection for the least concentrated samples. This limit may be extended by using a GMI magnetometer with a lower noise, for which one order of magnitude can be expected. ${ }^{12}$

\section{References and Notes}

1. S.-B. Seo, J. Yang, W. Hyung, E.-J. Cho, T.-I. Lee, Y. J. Song, H.-G. Yoon, J.-S. Suh, Y.-M. Huh, and S. Haam, Nanotechnology 18, 475105 (2007).

2. L. Li, D. Chen, Y. Zhang, Z. Deng, X. Ren, X. Meng, F. Tang, J. Ren and L. Zhang, Nanotechnology 18, 405102 (2007).

3. M. Tondra, A. Popple, A. Jander, R. L. Miller, N. Pekas, and M. D. Porter. J. Magn. Magn. Mater, 293, 725 (2005).

4. M. Kettering, J. Winter, M. Zeisberger, S. Bremer-Streck, H. Oehring, C. Bergemann, C. Alexiou, R. Hergt, K. J. Halbhuber, W. A. Kaiser and I. Hilger, Nanotechnology 18, 175101 (2007).

5. W. Weitschies, R. Kötitz, T. Bunte, and L. Trahms, Pharm. Pharmacol. Lett. 7, 1 (1997)

6. R. Kotitz, L. Trahms, H. Koch, and W. Weitschies, Biomagnetism: Fundamental Research and Clinical Applications, Elsevier Science Publishers, Amsterdam (1995).

7. F. Ludwig, S. Mäuselein, E. Heim, and M. Schilling, Rev. of Sci. Instr. 76, 106102 (2005).

8. K. Mohri, Mater. Sci. Eng. A185 141 (1994).

9. R. S. Beach and A.E. Berkowitz, Appl. Phys. Lett. 64, 3652 (1994).

10. G. V. Kurlyandskaya, M. L. Sánchez, B. Hernando, V. M. Prida, P. Gorria, and M. Tejedor, Appl. Phys. Lett. 82, 3053 (2003).

11. L. G. C. Melo, D. Ménard, A. Yelon, L. Ding, S. Saez, and C. Dolabdjian, J. Appl. Phys. 103, 033903 (2008).

12. L. Ding, S. Saez, C. Dolabdjian, L. Melo, A. Yelon, and D. Ménard, IEEE Sensors 9, 159 (2009).

13. E. Heim, S. Harling, F. Ludwig, H. Menzel, and M. Schilling. J. Phys. Condens. Matter 20, 204106 (2008).

14. F. Ludwig, E. Heim, S. Mauselein, D. Eberbeck, and M. Schilling, J. Magn. Magn. Mat. 293, 690 (2005).

15. A. Boukhenoufa, C.Dolabdjian, and D. Robbes, IEEE Sensors 5, 916 (2005). 